RESEARCH REPORT

\title{
EFFECT OF HYDROTHERAPY ON SPASTICITY AND GROSS MOTOR FUNCTIONS AMONG SPASTIC CEREBRAL PALSY CHILDREN
}

\begin{abstract}
OBJECTIVES

To determine the effects of hydrotherapy therapy on spastic cerebral palsy children using Modified ashworths scale and Gross motor functional classification scale after 32 weeks of training protocol.
\end{abstract}

\section{STUDY DESIGN}

Quasi experimental study

\section{STUDY SETTING}

Liaquat National Hospital

\section{PARTICIPANTS}

Spastic (diplegic, hemiplegic and quadriplegic) cerebral palsy children

\section{INTERVENTIONS}

58 Spastic Cerebral Palsywere assessed on the basis of modified Ashworth scale and gross motor function measure for spasticity and change in gross motor function respectively. Assessment was done, before and at the end of 10th week. Each therapy session was of 40-45 minutes thrice a week in a pool with the temperature maintained at $32^{\circ} \mathrm{C}-34^{\circ} \mathrm{C}$ and at the end cool down activities were performed.

\section{RESULTS}

Results indicated marked improvement in Muscle tone and Gross Motor Functioning of the enrolled children with $p=0.001$.

\section{CONCLUSIONS}

It was concluded that pool therapy is effective in reducing spasticity and improving gross motor functions among CP.

\section{KEY WORDS}

Cerebral Palsy, Spasticity, Hydrotherapy, Gross Motor function measure (GMFM), Modified Ashworth scale, hemiplegic.

\author{
Zeenat Fatima \\ Occupational Therapist \\ Institute of Physical Medicine and \\ Rehabilitation(IPM\&R) \\ DUHS \\ zeenatfatima.zf@gmail.com
Yarmiyah Rashaquat
Occupational Therapist
Institute of Physical Medicine and
Rehabilitation (IPM\&R)Ojha
DUHS
yermia.rashaquat@gmail.com

[Fatima Z, Rashaquat Y. Effect of Hydrotherapy on Spasticity And Gross Motor Functions Among Spastic CP Children. Pak. J. rehabil. 2019;8(1):13-18] 


\section{INTRODUCTION}

Cerebral palsy is a neurological impairment of motor disorder, balance, movement disorder, and postural deformities that appear during infancy and early childhood, although muscle tone and posture related abnormalities becomes more prominent in later years ${ }^{1}$. Globally CP occurs 1.5 to more than 4 per 1000 live births while in Asia the prevalence of CP accounts 1.4 per 1000 live births ${ }^{2}$ but in Pakistan the prevalence is 1.2 per 1000 live births that is expected to be much less than globally due to the poor health care infrastructure and lack of reportig3 ${ }^{3}$. Spastic diplegia and hemiplegic are common type of physical impairment occurring in $\mathrm{CP}$ children presenting with the symptoms of impaired posture, balance,gait abnormalities, and coordination along with swallowing and speech difficulties.There is probability of decreased cognitive skills that interrupts their daily activities and community participation as per expectations of social norms ${ }^{4}$.

Major etiological factors causing the CP are related to prenatal, peri-natal and postnatal periods that are intrauterine pathologies, maternal complications and postnatal sequences especially among preterm neonates. Infection, gestational diabetes, asphyxia, birth injury during delivery or cerebrovascular attack (CVA), high fever, meningitis, or encephalitis damage to the central nervous system (CNS) up to the age of 2 years are associated with CP but exact cause is still unknown ${ }^{5}$. The new advancement in medical technology and upgraded obstetric healthcarehas reduced the incidences. Spasticity is the major concern in cerebral palsy that is resistance to passive stretch that restricts the joint motion and ultimately reduces the participation and overall functioning ${ }^{6}$. Spasticity in CP is assessed by Modified Ashworth scale that was created by Bohannon and Smith in 1987 scoring from 0 that is no increase in muscle tone to 4 that denotes affected part rigid in flexion or extension ${ }^{7}$ Whereas Gross motor function in $C P$ is assessed and evaluated by using Gross motor function measure (GMFM) scale- $66^{8}$. Multiple studies have focused on potential effect of physical therapy in the management of CP and the goals of treatment in CP children with spasticity mainly focus on preventing the disabilities through minimizing the impacts of impairments and improving gross motor functional skills". According to the "American academy of cerebral palsy and developmental medicine", cerebral palsy patient must engage for at least 150 minutes of physical activity in a week according to their capacity. It will assistin strengthening musculature, increases cardiopulmonary endurance that will facilitate to perform more advance gross motor skills and improve their social participation.,2018) ${ }^{10}$.

Multiple approaches in the physical therapy are being used in the rehabilitation of CP such as stretching, strengthening, Neuro-developmental technique (NDT), motor relearning programs in reducing the spasticity and improving functional level in $\mathrm{CP}^{11}$. During current practices, primary focus on improving the functional independency and motor skills, thus aquatic therapy is good approach in this regard ${ }^{12}$. Furthermore hydrotherapy is effective treatment mode due to buoyancy and hydrostatic properties of water. Buoyancy force is equal to the weight of the fluid displaced by the object. Hydrotherapy was initially used in the rehabilitation unit that has six main classifications related to pool therapy: aquatic physiotherapy, the Hall wick concept, swimming, exercises in water, water walking, and playingin water. According the hydrotherapy is an effective treatment option in CP due to its thermal and mechanical effects. Thermal effects relaxes the muscle and facilitates in reducing the spasticity while mechanical effects of water are sufficient in reducing the effect of gravity and minimizing the joint loading, furthermore, water provides enjoyable environment to perform multiple functional activities for cerebral palsy ${ }^{13}$. Exercises in the pool are always performed at the temperature of $33^{\circ}-350 \mathrm{C}$ that is essential in reducing the spasticity by relaxing the muscles ${ }^{14}$. Besides these effects hydrotherapy has effects on strengthening the muscles and endurance since water creates resistance during walking that strengthens the muscles ${ }^{15}$ Activities performed in water are easier and effective as compared to the land but it has a major safety issues that should be identified before the start of exercises for the better results ${ }^{16}$. Hydrotherapy has several positive effects in the management of spasticity, joint range of motion andimproving balance in CP.Since the aquatic exercise reduces spasticity thus has potential effects in improving the gross motor functions among CP.In other words exercising in water provides opportunities to perform functional activities with ease and these exercises are not only effective for spasticity but also beneficial for reducing the risk of fall and improve the balance and related abnormalities ${ }^{17}$. Several studies have been conducted in the favor of hydrotherapy but some authors emphasized oncombination of conventionaland aquatic therapy for better results during the rehabilitation of $\mathrm{CP}^{16}$. Hydrotherapy is not routinely being practiced in the rehabilitation units due to the unavailability of trained staff and customized exercise protocols. For the better results in the management of advance gross motor skills in $\mathrm{CP}$, hydrotherapy should be incorporated with traditional training programs with special measures under the trained physical therapists ${ }^{18}$. However the effects of hydrotherapy are least documented, and more researches are required to be conducted on hydrotherapy to evaluate its effects on spastic CP patients for basic gross functioning that will assist the therapist in better and time effective treatment approach. furthermore hydrotherapy needs to be 
combined with the traditional therapy and requires the trained staff and strategized protocol for the better results hence this study was conductedto determine the effects of pool therapy on spasticity in cerebral palsy children for better gross motor functioning.

\section{METHODOLOGY}

\section{Study Setting}

The study was conducted at Liaquat National hospital.

\section{Target Population}

Spastic diplegic and hemiplegic cerebral palsy children enrolled in physical therapy outpatient department.

\section{Study Design}

Quasi experimental study

\section{Sample Size}

Sample size was calculated using Open Epi version 3 software. A previous study conducted in 20147 Pediatric Aquatic Therapy on Motor Function and Enjoyment in Children diagnosed with Cerebral Palsy of Various Motor Severities was considered to calculate sample size. By keeping confidence level $95 \%$ and $5 \%$ bound of error and Sample size was calculated as $n=41$.

\section{Data Analysis Procedure}

Data was entered and analyzed on SPSS version 20 software Qualitative data was reported as frequency and percentages and quantitative data was reported as mean and standard deviation. Independent t-test was applied to see post treatment variable to analyze their mean difference.

\section{Sampling Technique}

Non probability convenient sampling was used

\section{Sample Selection Inclusion Criteria ${ }^{23}$}

Both Male and female aged 5-7 years

Spasticity grade from mild to moderate

Able to follow the instruction

No any genetic disorder

\section{Exclusion Criteria}

Mild to moderate cognitive impairment ${ }^{20}$

Open wound or infection ${ }^{20}$

Fixed contractures ${ }^{23}$

Previous limb surgeries within 6 months ${ }^{20}$

\section{Interventional Strategies}

After taking consent from parent/guardian, participants were included in intervention program recruited on the basis of Modified Ashworths Scale and Gross Motor and Functional Measure for spasticity and change in gross motor function respectively. Assessment was done twice in the study, before the start of session and at the end of 10th week of intervention. The participants were engaged in therapy session for 40-45 minutes thrice a week for 10 weeks that is 5-7 min warm up followed bt 30-35 minutes of conditioning exercises then ending with 5-7 min of cool down. Before starting the conditioning exercises, warm up of 5-7 minutes was performed by participants inclusive of walking on shallow water followed by conditioning exercises inclusive of static stretching ofsmall and large muscle group of upper and lower limb and strengthening of lower limbs using sand bags on IRM methods in a swimming pool with the temperature maintained at $28-31^{\circ} \mathrm{C}$ for the duration of 30-35 minutes and at the end Participants performed cool down of 5-7 minutes.

\section{Data Collection Procedure}

1) Modified Ashworths Scale

Modified Ashworth scale is a tool to assess the spasticity in CP scoring from 0 that is no increase in muscle tone to 4 that denotes affected part rigid in flexion or extension. It has excellent inter rater reliability of $(\mathrm{KW}=0.87)^{7}$.

\section{2) Gross Motor and Functional Measure scale-66} GMFM-66 is a reliable tool designed and evaluated the gross motor change over the time in cerebral palsy. It was first established in late 80 s and it has excellent reliability of ICC 0.898. GMFM-66 shortest version of the GMFM-88 that comprises 66 items divided into the 5 categories (lying and rolling, sitting, crawling and kneeling, standing, walking, running and jumping) and clinician evaluates the patient according to these domains.

\section{Ethical Consideration}

Informed consent was taken from the attendants of the children prior to the start of study.

Any information that was contained from this study was kept confidential.

The protection of the privacy of research participants was ensured

Data collection in the hospital was done with the permission of department head.

\section{RESULTS}

A total number of 41 participants were recruited in this study among that $56.14 \%$ were male and $43.86 \%$ were female as shown in the figure 1 The mean age of participants was 4.49.Type of cerebral palsy children included in this study shown in figure 2.

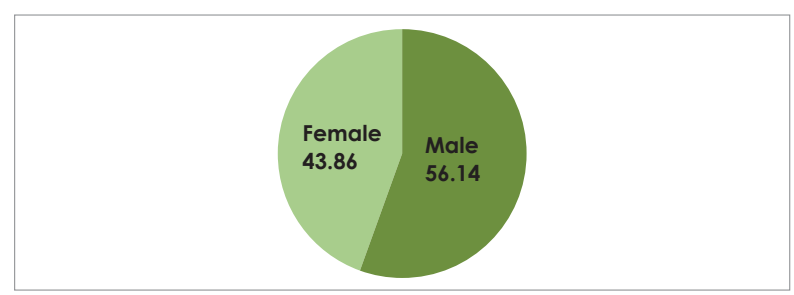

Figure 1. Presenting the percentage of male to female ratio. 


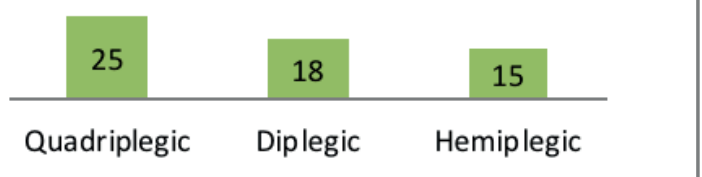

Figure 2. Represents Frequency of different types of cerebral palsy.

Overall improvement in muscle tone was observed among children with spastic cerebral palsy by using Modified Ashworth scale after hydrotherapy treatment.Region wise mean value of elbow, wrist, finger and thumb reduces before and after therapy in elbow(Pre=2.68, Post=1.86), wrist (Pre=2.53, Post=1.68), finger (Pre=2.63, Post=1.72), thumb (Pre=2.63, Post=1.77), (figure 3) and in lower limb improvement is shown in Hamstring (Pre=4.25, Post=3.01), Quadriceps (Pre=4.25. Post=3.15), Gastrocnemius (Pre=4.53, Post=3.32), Soleus (Pre=4.53, Post=3.31) showing significant improvement in muscle tone.

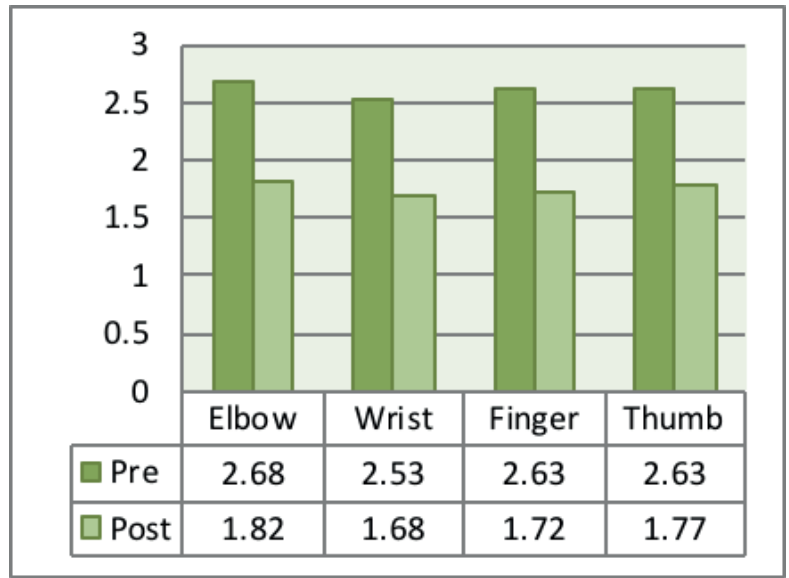

Figure 3. showing the significant improvement in muscle tone of upper limb.

Table 1 shows improvement in gross motor function

\begin{tabular}{|c|c|c|c|c|}
\hline Ability & $\begin{array}{c}\text { Pre } \\
\text { Mean } \\
\text { score }\end{array}$ & $\begin{array}{l}\text { Post } \\
\text { Mean } \\
\text { score }\end{array}$ & $\pm S D$ & $\begin{array}{c}P \\
\text { value }\end{array}$ \\
\hline \multirow[t]{2}{*}{$\begin{array}{l}\text { Lying \& } \\
\text { Rolling }\end{array}$} & 76.34 & 90.35 & \pm 15.04 & 0.0001 \\
\hline & 64.72 & 82.97 & \pm 12.75 & 0.0001 \\
\hline $\begin{array}{c}\text { Crawling } \\
\& \\
\text { Kneeling }\end{array}$ & 59.74 & 68.38 & \pm 6.1 & 0.011 \\
\hline Standing & 14.78 & 32.73 & \pm 16.74 & 0.001 \\
\hline $\begin{array}{l}\text { Walking, } \\
\text { Running } \\
\& \\
\text { Jumping }\end{array}$ & 9.77 & 21.48 & \pm 11.86 & 0.001 \\
\hline $\begin{array}{l}\text { Total } \\
\text { Score }\end{array}$ & 45.07 & 58.38 & \pm 12.48 & 0.0001 \\
\hline
\end{tabular}

on GMFM scale after treatment in different domains that is lying and rolling $(P=0.0001)$, sitting $(P=0.0001)$, crawling andkneeling $(P=0.011)$, standing, walking \& jumping $(P=0.0001)$.

\section{DISCUSSION}

Hydrotherapy is beneficial in the pediatric population and to furthers investigate these effects on cerebral palsy this study was conducted. Results of this study concluded that after 10 weeks of treatment given to the patient showed significant improvement in the muscle tone of the both upper and lower limbs. Chiu in his study has also concluded that warm water relaxes the muscle and reduces the spasticity. Notably, hydrotherapy had positive effects on gross motor function when assessed on 66-item GMFM scale ${ }^{19}$. According to the Fragala, activities performed in water are motivating and thus facilitates the patients to perform more functional activities that lead to the improved community participations in CP patient having impaired mobility ${ }^{20}$.

According to Wanees Badaway Spastic CP is the condition that is causing the postural problems that alters the mobility and gait pattern thus walking in water proves resistance and strengthens the musculature that help to improve balance hence minimizing the risk of fall in the patients.similarly he has also focused on the tools and assistive devices placed in the pool for therapeutic session.That ensured safety during the therapy sessions. The patients were facilitated according to the individual need such as railing, handrails, staircase, and chairlifts to perform functional activities more smoothly ${ }^{21}$. Furthermore chiu in his study also concluded aquatic therapy have positive effect in improving the gross motor functions in CP and facilitating the patient to perform more advance skills under the supervision of therapis ${ }^{18}$.

Furthermore hydrotherapy also promotes the enjoyment in children as compared to conventional land-based exercises that increases their physical activities and motivation "1. Runzunretaraker in his study has also concluded that 12 week of hydrotherapy treatment is effective to reduce the spasticity in $\mathrm{CP}$ child and environment of water facilitates in this regard by providing the relaxation ${ }^{5}$. Previous studies also suggest that hydrotherapy has positive effects in improving the muscle function and joint range of motion but the training protocols are not well defined. Also hydrotherapy consists of joyful and low weight bearing exercises that provides pain free activities by reducing the joint loading and must be incorporated in the traditional exercises. Henceexercising in water, children find the environment more playful and enjoyable than land-based activities and perceive the therapy as play rather than exercise ${ }^{23}$. In contrast to this, 
multiple researchers have contradictory results that hydrotherapy is not an effective and convenient treatment approach in the rehabilitation of the CP because it requires trained staff, strategized training protocols and more caution while engaging the patient in the hydrotherapy pool, as there are more safety issues thus must be handled with care and expertise hence this study showed positive results for hydrotherapy in maintaining the spasticity and gross motor functional skills in spastic CP finally concluded that hydrotherapy is effective treatment protocol for CP patients and it has no any side effects ${ }^{24}$. Hutzler's study concluded that hydrotherapy is good optionsfor management of gross motor function in the patients who have neuromotor impairment with restrict abilities to perform gross motor and fine motor skills because it eases performance of various activities without getting fatigue and irritationin children as children enjoy water activities and exercising in pooll5. These outcomes suggest hydrotherapy has positive effects on management of cerebral palsy as compared to the land-based exercises and need further researches to establish this fact. However hydrotherapy is being used in most of the rehabilitation programs and thus gaining popularity nowadays, but there is limited literature supporting the hydrotherapy as a primary option in the rehabilitation of CP for spasticity, impaired balance, postural disorders, movement impairment and gait abnormality. Hence this creates the need for future studies on hydrotherapy to evaluate the effects of pool therapy on cerebral palsy patients. However there is some limitation in the study due to the small sample size therefore experimental studies are needed to determine the effectiveness of intervention. However convenient sampling created the biasness among the participants. Besides these limitations, significant effects of pediatric hydrotherapy on muscle tone and gross motor function was observed among spastic cerebral palsy.

\section{CONCLUSION}

Pediatric hydrotherapy suggested greater improvement in gross especially in spastic diplegic cerebral palsy as measured on GMFM scale. In addition to this hydrotherapy has a significant effect in improving the muscle tone that reduces the spasticity grade in CP children. This study suggest pediatric hydrotherapy is safe and effective mode of treatment and further studies should be conducted with large sample size and follow up and also should be compared with land-based exercises for better results.

\section{REFERENCES}

[1] Ryu K, Ali A, Kwon M, Lee C, Kim Y, Lee G, Kim J. Effects of assisted aquatic movement and horseback riding therapies on emotion and brain activation in patients with cerebral palsy. J PhysTher
Sci. 2016;28(12):3283-7.

[2] Stavsky M, Mor O, Mastrolia SA, Greenbaum S, Than NG, Erez O. Cerebral Palsy-Trends in Epidemiology and Recent Development in Prenatal Mechanisms of Disease, Treatment, and Prevention. Frontiers in pediatrics. 2017 13; 5:21.

[3] Palsi PÇ. Cerebral Palsy in Pakistani Children: A Hospital Based Survey.

[4] Lai CJ, Liu WY, Yang TF, Chen CL, Wu CY, Chan RC. Pediatric aquatic therapy on motor function and enjoyment in children diagnosed with cerebral palsy of various motor severities.J. Child Neurol. 2015;30(2):200-8.

[5] Dimitrijević L, Aleksandrović M, Madić D, Okičić T, Radovanović D, Daly D. The effect of aquatic intervention on the gross motor function and aquatic skills in children with cerebral palsy. Journal of human kinetics. 2012; 32:167-74.

[6] El-Shamy SM. Effects of antigravity treadmill training on gait, balance, and fall risk in children with diplegic cerebral palsy. Am J Phys Med Rehabil 2017 Nov 1;96(11):809-15.

[7] Charalambous CP. Interrater reliability of a modified Ashworth scale of muscle spasticity. InClassic papers in orthopaedics 2014 (pp. 415-417). Springer, London.

[8] Avery LM, Russell DJ, Rosenbaum PL. Criterion validity of the GMFM-66 item set and the GMFM-66 basal and ceiling approaches for estimating GMFM-66 scores. Developmental Medicine \& Child Neurology. 2013 Jun;55(6):534-8.

[9] Ammann-Reiffer C, Bastiaenen $\mathrm{CH}$, Meyer-Heim $A D$, van Hedel HJ. Effectiveness of robot-assisted gait training in children with cerebral palsy: a bicenter, pragmatic, randomized cross-over trial (PeLoGAIT). BMC pediatrics. 2017;17(1):64.

[10] Lang TC, Fuentes-Afflick E, Gilbert WM, Newman TB, Xing G, Wu YW. Cerebral palsy among Asian ethnic subgroups. Pediatrics. 2012 ; 129(4):e992.

[11] Dimitrijević L, Aleksandrović M, Madić D, Okičić T, Radovanović D, Daly D. The effect of aquatic intervention on the gross motor function and aquatic skills in children with cerebral palsy.Jhumkinet. 2012;32:167-74.

[12] Declerck M, Feys H, Daly D. Benefits Of Swimming For Children With Cerebral Palsy: A Pilot Study. J Sports Sci Med. 2013;7(2).

[13] Getz $M$, Hutzler $Y$, Vermeer A, Yarom $Y$, Unnithan $V$. The effect of aquatic and land-based training on the metabolic cost of walking and motor performance in children with cerebral palsy: A pilot study. ISRN Rehabilitation. 2012; 2012.

[14] Adar S, Dündar Ü, Demirdal ÜS, Ulaşı AM, Toktaş $H$, Solak Ö. The effect of aquatic exercise on spasticity, quality of life, and motor function in cerebral palsy. Turkish Journal of Physical Medicine and Rehabilitation. 2017 Jun;63(3):239.

[15] Khalaji M, Kalantari M, Shafiee Z, Hosseini MA. The effect of hydrotherapy on health of cerebral palsy patients: An integrative review. Iranian Rehabilitation Journal. 2017;15(2):173-80. 
[16] Adar S, Dündar Ü, Demirdal ÜS, Ulaşlı AM, Toktaş $\mathrm{H}$, Solak Ö. The effect of aquatic exercise on spasticity, quality of life, and motor function in cerebral palsy. TURK J PH MED REHAB(2587-0823). 2017; 63(3).

[17] Jorgić B, Dimitrijević L, Aleksandrović M, Okičić T, Madić D, Radovanović D. The swimming program effects on the gross motor function, mental adjustment to the aquatic environment, and swimming skills in children with cerebral palsy: A pilot study. Specijalnaedukacija i rehabilitacija. 2012; $11(1): 51-66$.

[18] CChiu HC, Ada L, Lee SD. Balance and mobility training at home using Wii Fit in children with cerebral palsy: a feasibility study. BMJ open. 2018;8(5):e019624.

[19] RRoostaei $M$, Baharlovei $H$, Azadi $H$, Fragala-Pinkham MA. Effects of aquatic intervention on gross motor skills in children with cerebral palsy: a systematic review. Physical \& occupational therapy in pediatrics. 2017; 37(5):496-515

[20] BBADAWY WM, Ibrahem MB. Comparing the effects of aquatic and land-based exercises on balance and walking in spastic diplegic cerebral palsy children. Med. J. Cairo Univ. 2016; 84(1).
[21] Jorgic B, Aleksandrović $M$, Dimitrijević $L$, Živković D, Özsari M, Arslan D. The effects of a program of swimming and aquatic exercise on flexibility in children with cerebral palsy. FactaUniversitatis, Series: Physical Education and Sport. 2014:71-82.

[22] Maniu DA, Maniu EA, Benga I. Effects of an aquatic therapy program on vital capacity, quality of life and physical activity index in children with cerebral palsy. Human \& Veterinary Medicine. 2013; 5(3).

[23] Olama KA, Kassem HI, Aboelazm SN. Impact of aquatic exercise program on muscle tone in spastic hemiplegic children with cerebral palsy. Clin Med (Northfield II) 2015; 1 (4):138-44.

[24] Maniu DA, Maniu EA, Benga I. Effects of an aquatic therapy program on vital capacity, quality of life and physical activity index in children with cerebral palsy. Human \& Veterinary Medicine. 2013;5(3).

[25] Roostaei $M$, Baharlovei $H$, Azadi $H$, Fragala-Pinkham MA. Effects of aquatic intervention on gross motor skills in children with cerebral palsy: a systematic review. Physical \& occupational therapy in pediatrics. 2017;37(5):496-515. 